\title{
Increase in Critical Current Density for Increasing Applied Magnetic Field in a High-Temperature Superconducting Superlattice
}

\author{
Shinichi Ishiguri ${ }^{1}$, Shotaro Tawara ${ }^{2}$ \\ Nihon University ${ }^{1}$, Fukui National College of Technology ${ }^{2}$ \\ 1-2-1 Izumi-Cho, Narashinoshi, Chiba 275-8575 JAPAN \\ TEL: +81-47-474-9143
}

Email: S. Ishiguri: ishiguri.shinichi@nihon-u.ac.jp, S. Tawara: 122646816st@gmail.com

\begin{abstract}
In the present work, a superlattice structure comprising superconducting and insulator layers is studied. Here, if a magnetic field is applied parallel to the layers, the lack of a pinning center leads to a novel transition; in particular, as the applied magnetic field is reduced, the stationary wave surrounding the magnetic flux quantum in the superconducting layer eventually collides with the superconductinginsulating interfaces on both sides because its radius becomes larger than the width of the superconducting layer. At this instant, the stationary wave will collapse, and a transition will occur: the magnetic quanta are collapsed and thus the uniform magnetic field distribution is achieved, which corresponds to the transition from the superconducting state to the normal state over critical current. Considering a one-dimensional model of the structure, a critical current density equation is derived that indicates an increase in the critical current density for increased applied magnetic field. Subsequently, the same calculation was conducted after changing the direction of the magnetic field component, and the combination of these two calculations expresses the anisotropic property of the structure. The phenomenon is also predicted for anisotropic critical current density. This phenomenon is an important discovery that helps manufacture high-temperature superconducting tape as well as large high-temperature superconducting coils.
\end{abstract}

Keywords: superlattice, critical current density, stationary wave, magnetic flux quantum, HTS coil

\section{Introduction}

Recently, high-temperature superconductors including Fe-based materials have been investigated thoroughly [1-12]. Discussions of these superconductors have mainly centered on critical quantities [13] such as temperature or current density. Research on the magnetic flux quanta and the trends relating to the critical current density are important; in general, critical current density depends on the magnitude and angle of the applied magnetic field at constant temperature. To obtain equations describing such dependence, fitting methods have been employed that consider the dynamics of the 
magnetic flux quanta. For this purpose, pinning forces under various magnetic fields and flux angles have been measured, and equations have been derived through theoretical statistics [14]. In general critical current density behaves in conventional HTS tape wire as follows: if a magnetic field is applied to the tape, the radius of the stationary wave surrounding the pinning center becomes smaller to maintain the value of $h / 2 e$. Thus, with the application of the magnetic field, the radius of the stationary wave will eventually be equal to that of the pinning center, at which point the magnetic flux quantum collapses, and a superconduction transition occurs. The value of the current density when this occurs is the critical current density. If larger magnetic fields are applied, the radius of the stationary wave becomes smaller. This trend implies that the critical current density decreases as the applied magnetic field increases.

Note that a Cooper pair surrounding a magnetic flux quantum contributes to the stationary wave. However, as described below, this stationery wave and its Cooper pair are destroyed at the transition from the superconducting state to the normal state, such that the material's magnetic field distribution must become uniform.

In our previous article [13], we proposed a transition concept under an applied magnetic field where the transition is defined as the point at which the stationary wave surrounding the flux quantum vanishes with the destruction of a Cooper pair, i.e., wherein the radius of the stationary wave decreases until it is equivalent to that of the pinning potential and circular motion ceases, thus resulting in the destruction of Cooper pairs. In that concept, increasing the applied magnetic field decreases the radius of the stationary wave such that the value of the flux quantum $(h / 2 e)$ remains constant, and the density of flux quanta in the sample increases. Based on the proposed transition concept and the wave function of the Cooper pair around the magnetic flux quantum, the wave function of an electron at the transition is obtained, and the critical current density is in turn obtained from this wave function in the transition. To account for anisotropic properties, the calculation was performed for the magnetic field components perpendicular and parallel to the two-dimensional current sheet. The calculated current densities for each case were combined, and the critical current density was calculated. Theoretical data agreed with measured data [13].

In the present paper, a superlattice structure comprising superconducting and insulator layers is considered. In this structure, no pinning center will occur if a magnetic field is applied parallel to the layers. That is, when reducing the applied magnetic field, the stationary wave surrounding the magnetic flux quantum eventually collides with the insulating layers on both sides, because the radius becomes larger than the width of the superconducting layer. At that moment, the stationary wave will collapse, and a transition will occur. A one-dimensional model of the structure is considered, based on which an equation for the critical current density is derived. According to this equation, an increase in the applied magnetic field will result in an increase in the critical current density. This phenomenon remains even when anisotropic critical current density is considered. Applying this phenomenon in 
HTS wires, we are able to manufacture larger HTS coils with more turns that consequently generated larger magnetic fields, resulting in larger critical current. That is, the larger the HTS coil we obtain, the higher the performance gets.

\section{Theory}

\subsection{A transition concept assuming the superlattice}

As shown in Fig. 1, this study is based on a superlattice structure comprising insulating and superconducting layers. The superconducting layers correspond to two-dimensional current sheets. In this study, material is assumed to be a Bi-2223 at $77 \mathrm{~K}$. When the width of the superconducting layers is sufficiently smaller than typically about $50 \mathrm{~nm}$, we assume that no pin exists. Thus, each flux quantum is weakly fixed by the repulsive force from the neighboring flux quanta. When a strong magnetic field is applied, the number of magnetic flux quanta increases because the radius of each magnetic flux quantum decreases [13] to maintain the constant value of each quantum constant, $h / 2 e$. In this study, we assume that the radius of a magnetic flux quantum corresponds to that of the stationary wave surrounding the quantum. Note that the stationary wave here implies the Cooper pair motion surrounding a magnetic flux quantum.

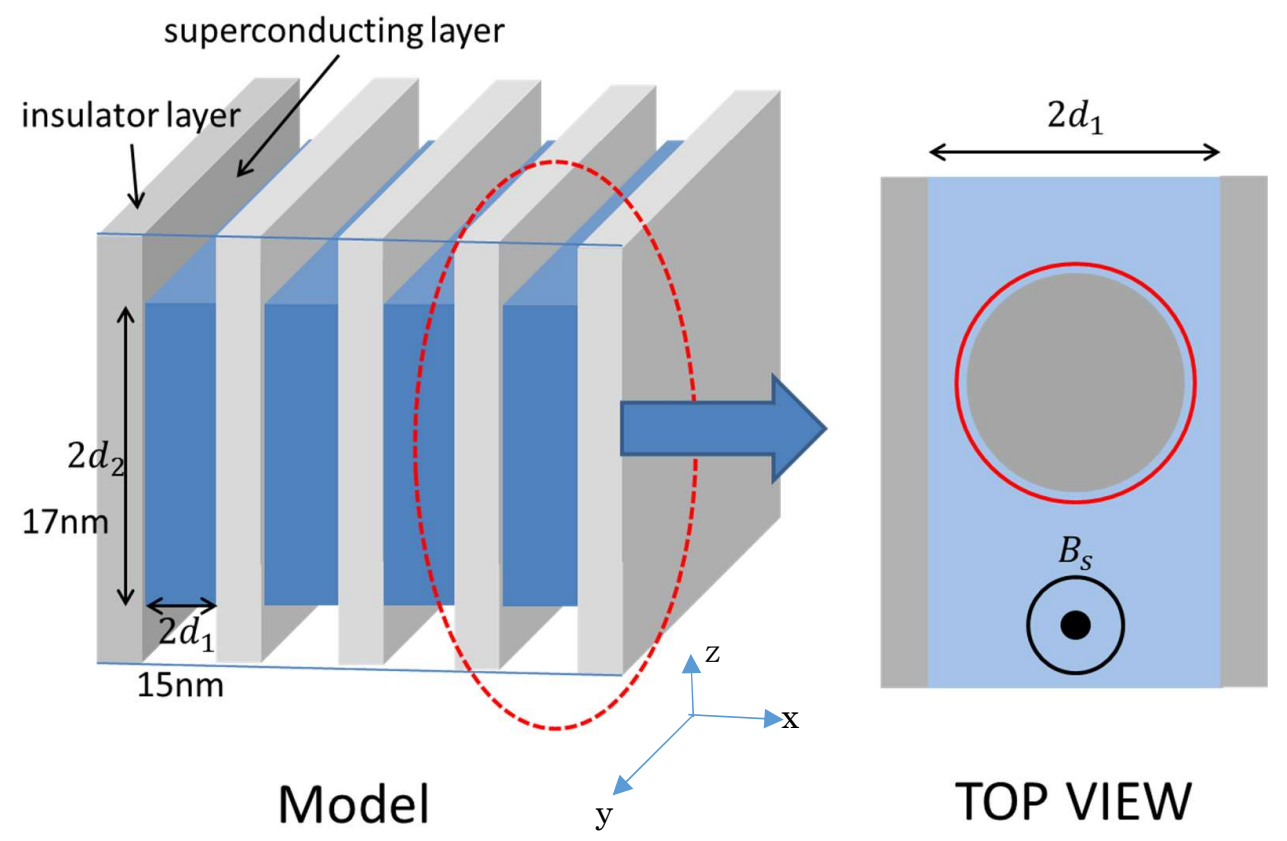

Fig. 1 Model of the superlattice featuring superconducting and insulating layers. The magnetic field, $\mathrm{B}_{\mathrm{s}}$, is applied parallel to the $\mathrm{z}$-axis. 
As described previously [13], the radius of the stationary wave surrounding a magnetic flux quantum varies with the magnitude of the applied magnetic field such that the value of the magnetic flux quantum is maintained at $h / 2 e$. That is, the application of a larger magnetic field results in a decrease in the radius of the stationary wave. As shown in Fig. 1, we apply a magnetic field parallel to the layers, i.e., along the z-axis. When the magnitude of this field is decreased, the radius of the stationary wave increases until eventually touching the insulator layers. At this point, the stationary wave collapses and a magnetic transition occurs, as shown in Fig. 2. This transition indicates the process of the magnetic flux quanta in the material collapse and thus achieve uniform magnetic field distribution, which is the distribution of the normal state: this contradicts the conventional HTS models.

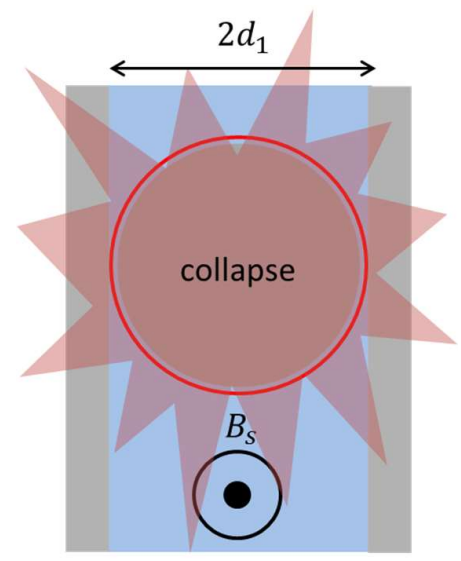

\section{TOP VIEW}

Fig. 2 Schematic of the magnetic transition. As a result of the stationary wave ceasing, the magnetic flux quantum also collapses.

\subsection{Critical current density under a magnetic field applied parallel to the layers}

In this study, cylindrical coordinates are employed. The wave function of the Cooper pair distributed along the magnetic flux quantum, i.e., the wave function related to the stationary wave surrounding the magnetic flux quantum [15] as follows.

$$
\Phi=|\Phi| \exp (j \varphi),
$$

with 


$$
\begin{aligned}
& \varphi=\oint \frac{q \vec{A}}{\hbar} \cdot d \vec{r}, \\
& \varphi=2 \mathrm{n} \pi,
\end{aligned}
$$

where $q, j, A$, and $n$ denote the charge of the pair, an imaginary unit, the vector potential, and an integer, respectively.

Magnetic flux quanta is derived as follows [15]:

$$
\int B \cdot d \vec{S}=\int \operatorname{rot} \vec{A} \cdot d \vec{S}=\oint \vec{A} \cdot d \vec{r}=\left(\frac{h}{2 e}\right) n .
$$

Equation (2) indicates that the wavenumber is given by

$$
\mathrm{k}=\frac{q A}{\hbar} \text {. }
$$

Henceforth, we change the $x-y$ coordinates as shown in Fig. 3.

In general, circular motion implies the center-of-mass motion of a Cooper pair. However, near the magnetic transition, the critical current comprises pair-broken normal electrons because the Cooper pair's kinetic energy does not contribute to the critical current. Thus, at the transition, the wave functions of electrons are considered and not that of the Cooper pairs. That is, a Cooper pair surrounding a magnetic flux quantum is broken at the transition. Accordingly, the wavenumber is halved at the transition:

$$
k_{e}=\frac{e|\vec{A}|}{2 \hbar}
$$

At the transition, the stationary wave should disappear, and thus, a wave function during the transition must be a decaying function in which the electron wavelength must be conserved. Therefore, the wave function of an electron at the transition is as follows:

$$
\psi=|\psi| \exp \left(-\frac{1}{\lambda} x\right)
$$

where $\lambda$ denotes the wavelength of an electron.

In this study, wavelength is the inverse of the wavenumber, so the wave function can be rewritten as

$$
\psi=|\psi| \exp \left(-\frac{e|\vec{A}|}{2 \hbar} x\right)
$$

Furthermore, we can consider the generation of an opposite-direction electron as a result of the destruction of circular motion:

$$
\psi=|\psi| \exp \left(-\frac{e|\vec{A}|}{2 \hbar}(-x)\right)
$$

As shown in Fig. 3, the scale of the potential distribution along the y-direction can be assumed to be infinitely large if we compare the scale of x-direction. Thus, it is not necessary to consider the motion along the y-direction. When considering the vector potential $\boldsymbol{A}=\left(A_{x}, A_{y}\right)$, only the motion associated with the $x$-component, $A_{\mathrm{x}}$, is affected by the force from the potential (i.e., the insulating layer). This implies that the decaying function can be considered only in a single dimension along the $x$-axis. Thus, 
when considering a superconducting layer, the decaying functions along both positions of $-\mathrm{x}_{0}$ and $+\mathrm{x}_{0}$ are superposed. The wave function at the transition is

$$
\begin{aligned}
\psi & =|\psi|\left\{\exp \left(-\frac{e}{2 \hbar} A_{x} x_{0}\right)+\exp \left(-\frac{e}{2 \hbar} A_{x}\left(-x_{0}\right)\right)\right\}, \\
\psi & =2|\psi| \cosh \left(\frac{e}{2 \hbar} A_{x} x_{0}\right) .
\end{aligned}
$$

Thus,

$$
\begin{aligned}
\psi & =2|\psi| \cosh \left(\frac{e}{2 \hbar} B_{s} d_{1} x_{0}\right), \\
\psi & =2|\psi| \cosh \left(\frac{e}{2 \hbar} B_{S} d_{1}^{2}\right) .
\end{aligned}
$$

The critical current density can therefore be expressed as:

$$
j_{c s}=e n v=4 e v|\psi|^{2} \cosh ^{2}\left(\frac{e B_{S}}{2 \hbar} d_{1}^{2}\right) .
$$

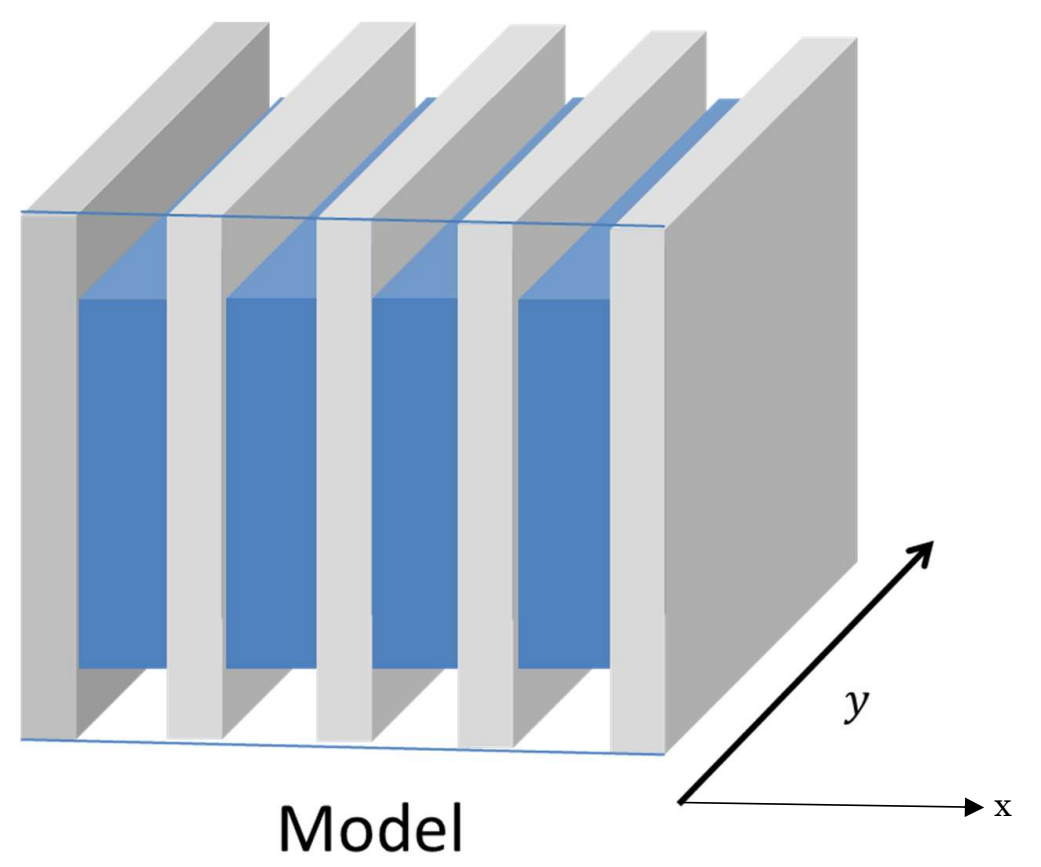

Fig. $3 \mathrm{x}$ - and y-axis direction of the superlattice model

2.2 Critical current density for magnetic field component applied perpendicular to the superlattice layers

Note that the direction of the magnetic field perpendicular $\mathrm{B}_{\mathrm{t}}$ is along the $\mathrm{x}$-axis. That is, along this direction, there is no structure of the superlattice of superconducting and insulator layers. Thus, the 
transition due to the stationary wave contacting both insulating-superconducting interfaces does not occur under the application of a perpendicular magnetic field. As the applied $\mathrm{Bt}$ is increased, the radius of the stationary wave loop decreases until it becomes equal to the coherence of Cooper pair that creates the stationary wave around the magnetic flux quantum. Note that the radius of the stationary wave in general is the coherence of the Cooper pair since the superlattice structure rules out the presence of pins. Although the radius of the pinning potential is replaced with the coherence here, this type of transition is similar to that described in [13]. A diagram showing the stationary wave occurring under applied $B_{\mathrm{t}}$ is shown in Fig. 4. As shown, this wave is 2-dimensional, while $B_{s}$ is one-dimensional.

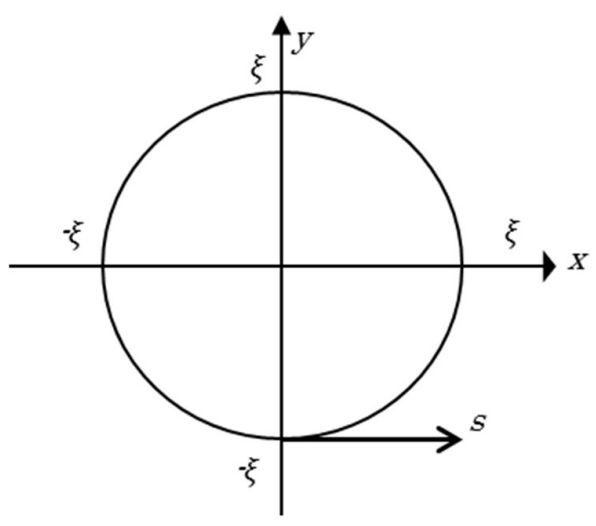

Fig. 4 Introduction of variable $s$

The variable $s$ is introduced is introduced in Figure 4. This variable is started at the circumference and is parallel to the r-direction. The wave function for the transition of an electron in this case is given by equation (14) as follows:

$$
\psi=|\psi| \exp \left(-\frac{1}{\lambda} s\right)
$$

From equations (6) and (14),

$$
\psi=|\psi| \exp \left(-\frac{e|\vec{A}|}{2 \hbar} s\right)
$$

Moreover, because the $x$ - and $y$-axes are arbitrarily defined, the $s$-axis can be made to correspond to the $x$-direction:

$$
A=B_{t} s .
$$

Since the variable $s$ is equal to the coherence $\xi$, the wave function becomes:

$$
\psi=|\psi| \exp \left(-\frac{e B_{t}}{2 \hbar} \xi^{2}\right) .
$$

Thus, 


$$
j_{c t}=|\psi|^{2} \operatorname{evexp}\left(-\frac{e \xi^{2}}{\hbar} B_{t}\right)
$$

where $v$ and $e$ denote drift velocity and electric charge.

\subsection{Anisotropy of the critical current density}

The net critical current density is the sum of the current densities associated with components $B_{\mathrm{s}}$ and $B_{\mathrm{t}}$. Thus, from equations (13) and (18),

$$
j_{c}=4 e v|\psi|^{2}\left\{\cosh ^{2}\left(\frac{e B_{s}}{2 \hbar} d_{1}^{2}\right)+\frac{1}{4} \exp \left(-\frac{e \xi^{2}}{\hbar} B_{t}\right)\right\} .
$$

\section{Result}

In Equation (19), $|\psi|^{2}$ and $\mathrm{v}$ are derived from [13] and the coherence length, $\xi$, is assumed to be 1 $\mathrm{nm}$. As shown in Fig. 5-1, the critical current density, $\mathrm{j}_{\mathrm{c}}$, increases with the magnitude of the applied magnetic field. That is, the expression from the magnetic component $B_{s}$ and $B_{t}$ istransformed to the magnetic field magnitudes and angles. To get more visibility in Fig. 5-2, this result is presented as a 3-dimensional graph.

Figs. 6 and 7 present the critical currents under the application of $B_{\mathrm{t}}$ and $B_{\mathrm{s}}$, respectively. As shown, the critical current undergoes a net increase under the application of $B_{\mathrm{s}}$. This results in the data shown in Figure 5-1. 


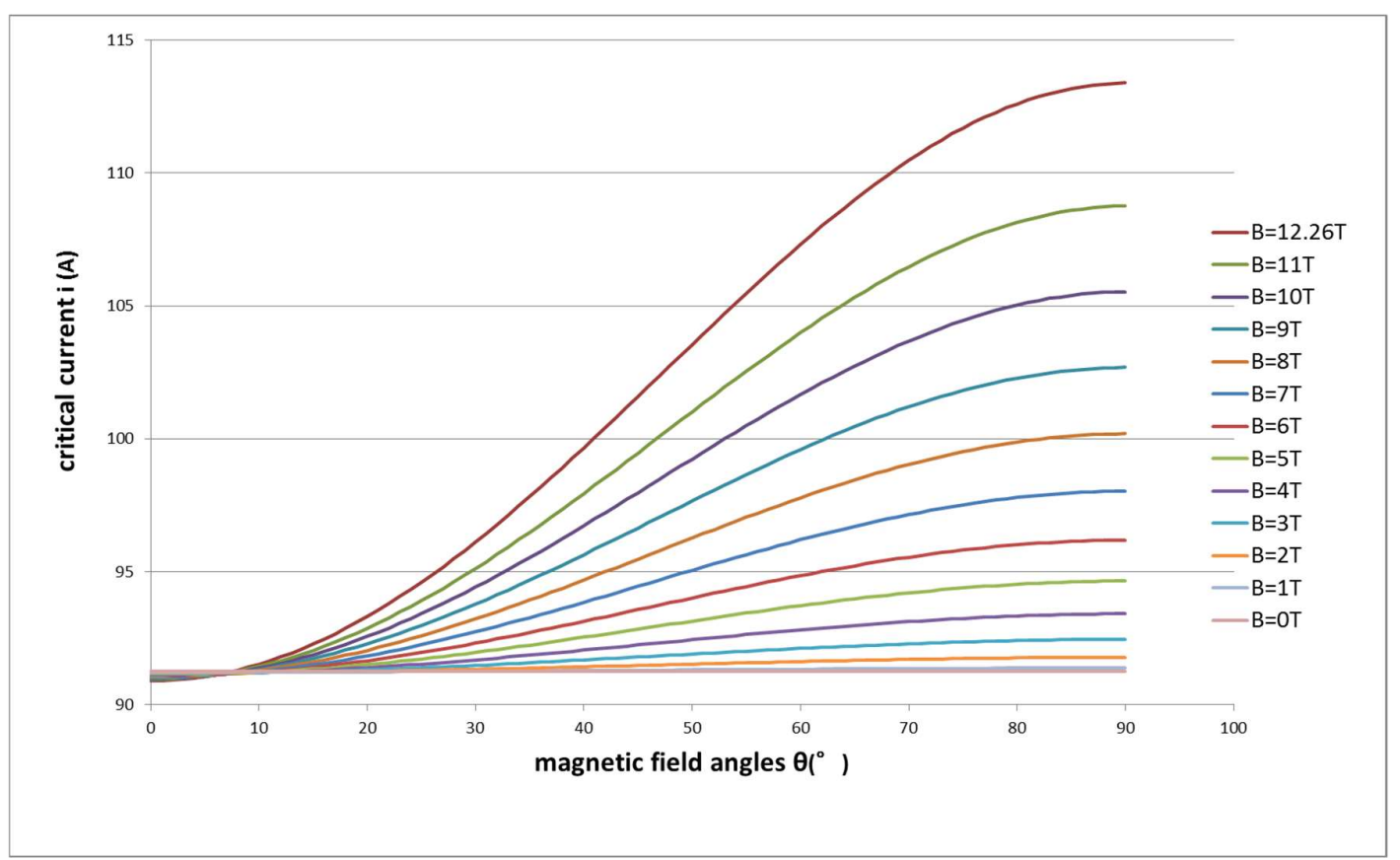

Fig. 5-1 Dependence of critical current on the applied magnetic field angle for different magnetic field magnitudes (first version)

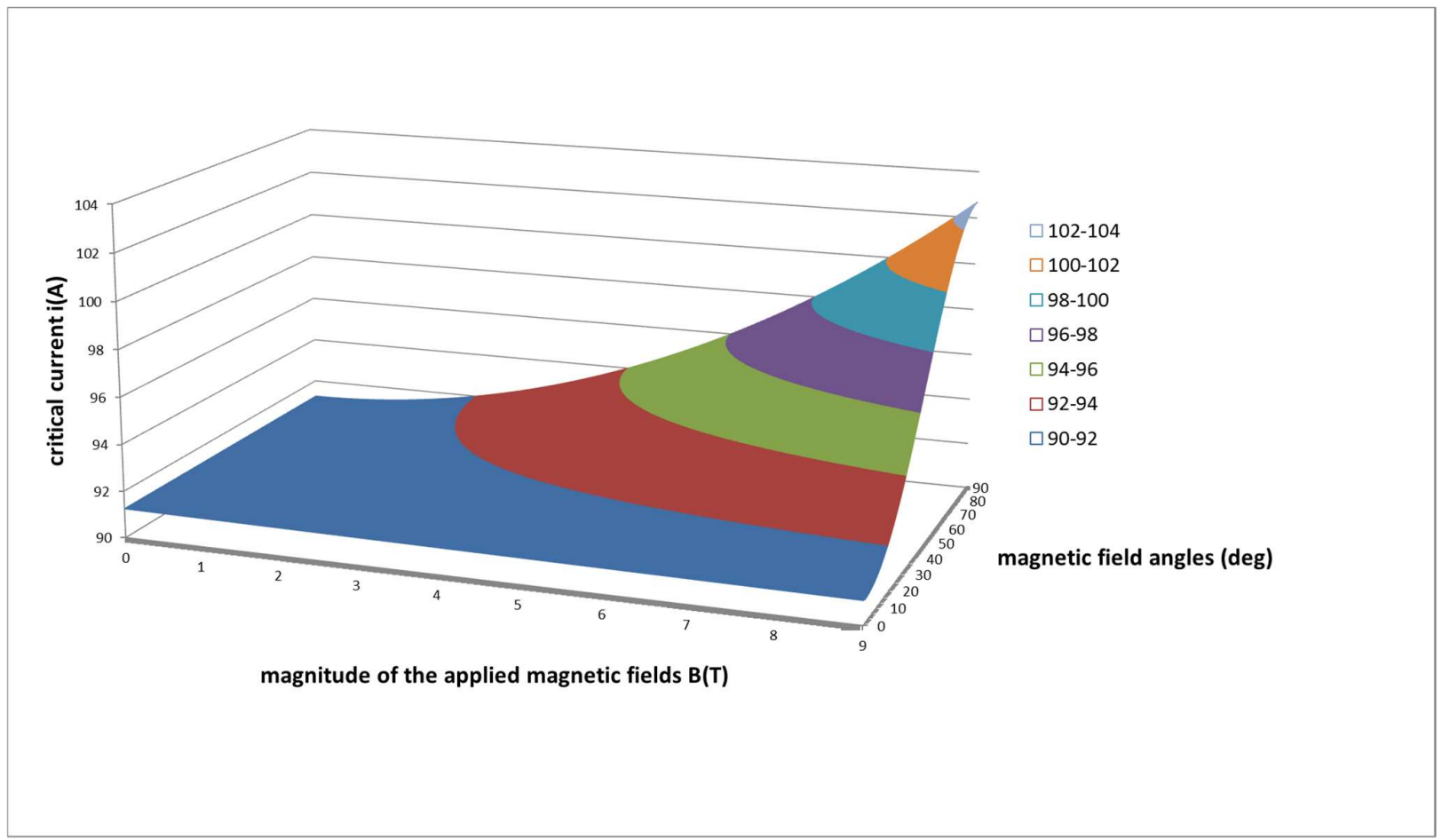

Fig. 5-2 Three-dimensional representation of the dependence of critical current upon magnetic field magnitude and angle (second version) 


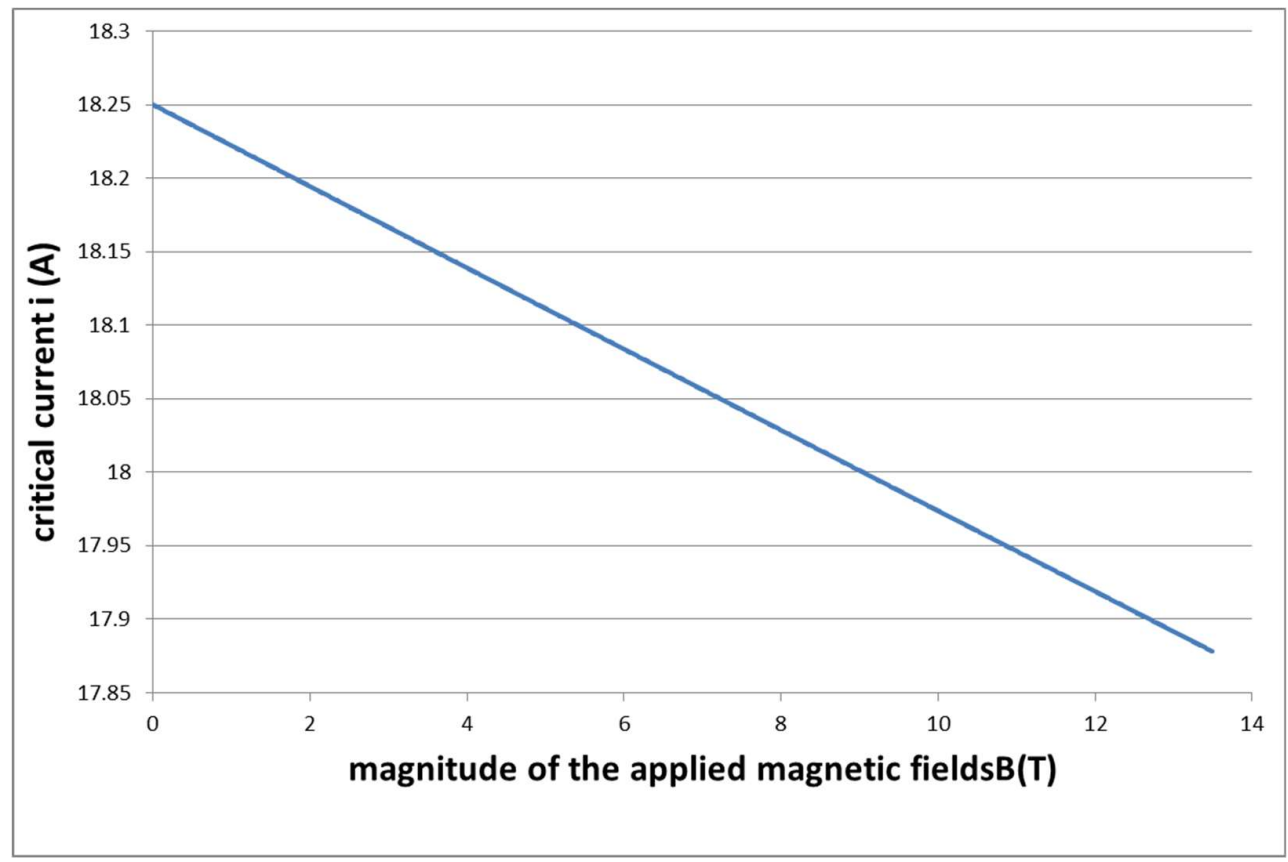

Fig. 6 Magnetic field dependence of the $B_{t}$ component (second term of equation (19)) of the critical current density jc.

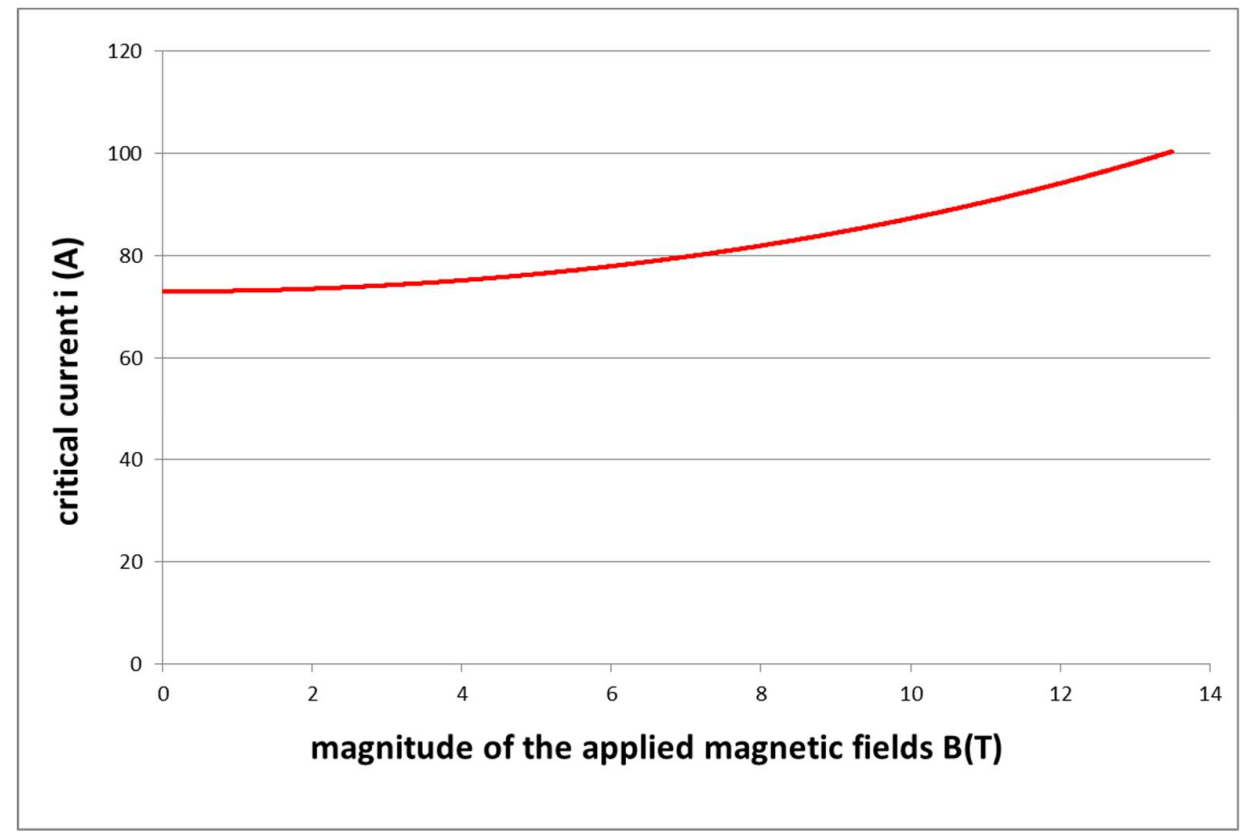

Fig. 7 Magnetic field dependence of the first term of equation (19) concerning $B_{s}$ 


\section{Discussion}

The main goal of this study was to improve the performance of a high-temperature superconducting (HTS) coil by manufacturing a coil made from a wire with a superlattice structure. Due to the high conductivity of the wire, a larger HTS coil (i.e., many turn coil) can be driven because the lager the HTS coil becomes, the lager magnetic field generation. That is, the critical current becomes higher, and as such the costs of a large HTS coil could likely be reduced. Thus, an application of the results of this study is the performance improvement of HTS coil applications. It is more efficient to prepare a larger HTS coil if the current density of the wire is higher.

In brief, the critical current density behaves in conventional HTS tape wire as follows: if a magnetic field is applied to the tape, the radius of the stationary wave surrounding the pinning center becomes smaller to maintain the value of $h / 2 e$. Thus, with the application of the magnetic field, the radius of the stationary wave will eventually be equal to that of the pinning center, at which point the magnetic flux quantum collapses, and the transition occurs. The value of the current density when this occurs is the critical current density. If larger magnetic fields are applied, the radius of the stationary wave becomes smaller. This trend implies that the critical current density decreases as the applied magnetic field increases.

However, as presented here, considering a superlattice structure results in a new phenomenon As shown in Fig. 5-1 and 5-2, critical current increases with the magnitudes of magnetic fields. Deriving from these figures, the higher the magnetic field, the greater the gradient of increase in critical current. Thus, many turn HTS coil wound by this superlattice exhibits high performances in terms of central magnetic fields and stored energy.

This phenomenon is reverse, compared with the conventional critical current density phenomenon.

\section{Conclusion}

In the present paper, a superlattice structure comprising superconducting and insulating layers is studied. If a magnetic field is applied parallel to the layers, the lack of a pinning center results in a transition concept; in particular, when the magnitude of the applied magnetic field is reduced, the stationary wave surrounding the magnetic flux quantum will eventually intersect the insultingsuperconducting interfaces on both sides; at this point, the stationary wave will collapse, and a transition will occur. The above transition is different in terms of the process for the conventional superconductors, but it describes universally the process of the magnetic flux quanta destructions and the process of material obtaining the uniform magnetic field distribution. This model can be considered 
in terms of a single dimension in order to derive a critical current density equation. When the equation is applied, it is found that increasing the magnitude of the applied magnetic field component results in an increase in the critical current density. The magnetic transition is predicted even when the anisotropic property of the critical current density is considered. This paper results leads to put into practice of large HTS coil. In general HTS coil is basic application for other applications such as MRI, Mglev, acceleration of elementary particles, and nuclear energy device.

\section{References}

[1] M. Yamaguchi, A. Honma,, et al: IEEE Transactions on Applied Superconductivity, VOL.13, No.2 , 1848 (2003)

[2]. S. Ishiguri, Results in Physics 3 74-79 (2013)

[3]. P. W. Anderson, et al, J. Phys.: Condens. Matter Vol. 16, No. 24, (2004) R755

[4]. P. A. Lee, et al, Rev. Modern Phys., Vol. 78, (2006) 17

[5]. M. Ogata, H. Fukuyama, Rep. Prog. Phys., Vol. 71, (2008)

[6]. M. Azuma, et al, Phys. Rev. Lett, 733463 (1994)

[7]. F. C. Zhang, T. M. Rice, Phys. Rev., Vol. B 37, (1988) 3759

[8] S. Uchida, Japanese Applied Physics (Institute Journal ), Vol. 80, No. 5, (2011) 383-386

[9] T. Fujita, J. Cryogenics and Superconducting Societies of Japan, Vol. 47, No. 2, (2012) 89-95

[10]. J. G. Bednorz and K. A. Müller, Zeitschrift für Physik B 64, 189 (1986)

[11]. J. Nagamatsu, et al, Nature 410, 63 (2001)

[12].Y. Kamihara, et al, J. Am. Chem. Soc. 128 (31), 10012-10013 (2006)

[13]. S. Ishiguri and T. Sato, Physica C 469 135-138 (2009)

[14] T. Kiss, M.Inoue, T. Kuga, M. Ishimaru, S. Egashira, S. Irie, T. Ohta, K. Imamura, M. Yasunaga, M. Takeo, T. Matsushita, Y. Iijima, K. Kakimoto, T.Saitoh, S. Awaji, K. Watanabe, Y. Shiohara: Physica C 392-369, 1053 (2003)

[15] M. Yamamura, M. Sugawara, O. Tsukamoto, M. Yamaguchi, M. Yamamoto, Technology of Superconductivity (Institute of electrical engineers of Japan) p. 25 (2001) 